\title{
On some inequalities for derivatives of algebraic polynomials in unbounded regions with angles
}

\author{
Cevahir Doğanay Gün
}

Gaziantep University, Gaziantep, Turkey, cevahirdoganaygun@ gmail.com, ORCID: 0000-0003-3046-7667

\section{A B S T R A C T}

In this work we study Bernstein-Walsh-type estimations for the derivative of an arbitrary algebraic polynomial in regions with interior zero and exterior non zero angles.

\section{ARTICLE INFO}

\section{Research article}

Received:

Accepted:

Keywords:

Algebraic polynomial,

quasicircle,

smooth curve,

inequalities.

*Corresponding author

\section{Introduction}

Let $\mathbb{C}$ denote the complex plane and $\overline{\mathbb{C}}:=\mathbb{C} \cup\{\infty\} ; G \subset \mathbb{C}$ be a bounded Jordan region with boundary $L:=\partial G$ such that $0 \in G$;

Let $\left\{z_{j}\right\}_{j=1}^{l}$ be the fixed system of distinct points on the curve $L$. We consider generalized Jacobi weight function $h(z)$ which is defined as follows:

$h(z):=\prod_{j=1}^{l}\left|z-z_{j}\right|^{\gamma_{j}}, \quad z \in \mathbb{C}$,

where $\gamma_{j}>-2$, for all $j=1,2, \ldots, l$.

Let $\wp_{n}$ denotes the class of all algebraic polynomials $P_{n}(z)$ of degree at most $n \in \mathbb{N}$.

Let $p>0$. For the Jordan region $G$, we introduce:

$$
\begin{aligned}
\left\|P_{n}\right\|_{p}:=\left\|P_{n}\right\|_{A_{p}(h, G)}:=\left(\iint_{G} h(z)\left|P_{n}(z)\right|^{p} d \sigma_{z}\right)^{1 / p}, 0<p<\infty, \\
\left\|P_{n}\right\|_{\infty}:=\left\|P_{n}\right\|_{A_{\infty}(1, G)}:=\max _{z \in \bar{G}}\left|P_{n}(z)\right|, p=\infty,
\end{aligned}
$$

and $A_{p}(1, G) \equiv A_{p}(G)$, where $\sigma$ be the two-dimensional Lebesgue measure.

When $L$ is rectifiable, for any $p>0$, let

$$
\begin{array}{r}
\left\|P_{n}\right\|_{\mathcal{L}_{p}(h, L)}:=\left(\int_{L} h(z)\left|P_{n}(z)\right|^{p}|d z|\right)^{1 / p}<\infty, 0<p<\infty, \\
\left\|P_{n}\right\|_{\mathcal{L}_{\infty}(1, L)}:=\max _{z \in L}\left|P_{n}(z)\right|, p=\infty,
\end{array}
$$

and $\mathcal{L}_{p}(1, L) \equiv \mathcal{L}_{p}(L)$.

Let us set $\Omega:=\overline{\mathbb{C}} \backslash \bar{G}=\operatorname{ext} L ; \Delta(w, R):=\{w:|w|>R, R>1\}, \Delta:=\Delta(0,1)$ and let $w=\Phi(z)$ be the univalent conformal 
mapping of $\Omega$ onto $\Delta$ such that $\Phi(\infty)=\infty$ and $\lim _{z \rightarrow \infty} \frac{\Phi(z)}{z}>0 ; \Psi:=\Phi^{-1}$. For $R>1$ we define $L_{R}:=\{z:|\Phi(z)|=R\}, G_{R}:=$ int $L_{R}, \Omega_{R}:=\operatorname{ext} L_{R}$.

Well known Bernstein -Walsh Lemma [26] says that:

$$
\left\|P_{n}\right\|_{C\left(\bar{G}_{R}\right)} \leq R^{n}\left\|P_{n}\right\|_{C(\bar{G})} .
$$

Analogous estimation with respect to the quasinorm (4) for $p>0$ was obtained in [19] for $h(z) \equiv 1$ (i.e., $\gamma_{j}=0$ for all $j=$ $1,2, \ldots, l)$ and in [8, Lemma 2.4] for $h(z) \neq 1$, defined as in (1) as following:

$$
\left\|P_{n}\right\|_{\mathcal{L}_{p}\left(h, L_{R}\right)} \leq R^{n+\frac{1+\gamma^{*}}{p}}\left\|P_{n}\right\|_{\mathcal{L}_{p}(h, L)}, \gamma^{*}=\max \left\{0 ; \gamma_{j}: 1 \leq j \leq l\right\}
$$

To give a similar estimation to (5) for the $A_{p}(h, G)$-norm, first of all we will give the following definition.

Definition 1. [20, p.97], [23] The Jordan arc (or curve) L is called $K$-quasiconformal ( $K \geq 1$ ), if there is a $\mathrm{K}$-quasiconformal mapping $\mathrm{f}$ of the region $\mathrm{D} \supset \mathrm{L}$ such that $\mathrm{f}(\mathrm{L})$ is a line segment (or circle).

Let $F(L)$ denote the set of all sense preserving plane homeomorphisms $f$ of the region $D \supset L$ such that $f(L)$ is a line segment (or circle) and let

$$
K_{L}:=\inf \{K(f): f \in F(L)\},
$$

where $K(f)$ is the maximal dilatation of $f$. Then $L$ is a quasiconformal curve, if $K_{L}<\infty$, and $L$ is a $K$-quasiconformal curve, if $K_{L} \leq K$.

A curve $L$ is called a quasiconformal, if it is a $K$-quasiconformal for some $K>1$.

The Bernstein-Walsh type estimates for the norm (2), for the regions with quasiconformal boundary and weight function $h(z)$, defined in (1) with $\gamma_{j}>-2$, for all $p>0$ as follows

$$
\left\|P_{n}\right\|_{A p\left(h, G_{R}\right)} \leq c_{1} R^{*}{ }^{n+\frac{1}{p}}\left\|P_{n}\right\|_{A p(h, G)^{\prime}}
$$

was found in [3] (see, also [2]), where $R^{*}:=1+c_{2}(R-1), c_{2}>0$ and $c_{1}:=c_{1}\left(G, p, c_{2}\right)>0$ constants, independent from $n$ and $R$. It's well known that quasiconformal curves can be non-rectifiable (see, for example, [16], [20, p.104]).

Analogous estimation was studied for $A_{p}(1, G)$-norm, $p>0$, for arbitrary Jordan region in [4, Theorem1.1] and for any $P_{n} \in$ $\wp_{n}, R_{1}=1+\frac{1}{n}$ and arbitrary $R, R>R_{1}$, was obtain

$$
\left\|P_{n}\right\|_{A_{p}\left(G_{R}\right)} \leq c R^{n+\frac{2}{p}}\left\|P_{n}\right\|_{A p\left(G_{R_{1}}\right)}{ }^{\prime}
$$

where $c=\left(\frac{2}{e^{p}-1}\right)^{\frac{1}{p}}\left[1+O\left(\frac{1}{n}\right)\right], n \rightarrow \infty$.

For a rectifiable quasiconformal curve $L, N$. Stylianopoulos [24] obtained the following estimate:

$$
\left|P_{n}(z)\right| \leq c \frac{\sqrt{n}}{d(z, L)}\left\|P_{n}\right\|_{A_{2}(G)}|\Phi(z)|^{n+1}, \quad z \in \Omega,
$$

where $d(z, L):=\inf \{|\zeta-z|: \zeta \in L\}$, a constant $c=c(L)>0$ depending only on $L$.

Analogous results of (7)-type for $\left|P_{n}(z)\right|$, different weight function $h$, unbounded region $\Omega$ were obtained in [17, p.418-428], [5], [6], [7], [8], [9], [10], [11], [15], [22] and others.

In this work, we study the pointwise estimations for the derivative $\left|P_{n}^{\prime}(z)\right|$ in unbounded region $\Omega$ with zero angles as the following type

$$
\left|P_{n}^{\prime}(z)\right| \leq c_{2} \eta_{n}(G, h, p, d(z, L),|\Phi(z)|)\left\|P_{n}\right\|_{p}, z \in \Omega,
$$

where $c_{2}=c_{2}(G, p)>0$ is a constant independent of $n, Z$ and $P_{n}$, and $\eta_{n}(G, h, p, d(z, L),|\Phi(z)|) \rightarrow \infty, n \rightarrow \infty$, depending on the properties of the $G, h$ and from the distance of point $z \in \Omega$ to the $\bar{G}$. 


\section{Definitions and main results}

Throughout this paper, $c, c_{0}, c_{1}, c_{2}, \ldots$ are positive and $\varepsilon_{0}, \varepsilon_{1}, \varepsilon_{2}, \ldots$ are sufficiently small positive constants (generally, different in different relations), which depends on $G$ in general and, on parameters inessential for the argument, otherwise, the dependence will be explicitly stated. For any $k \geq 0$ and $m>k$, notation $i=\overline{k, m}$ means $i=k, k+1, \ldots, m$.

Let $z=z(s), s \in[0$, mes $L]$ denote the natural representation of $L$.

Definition 2. We say that $L \in C_{\theta}$, if $L$ has a continuous tangent $\theta(z):=\theta(z(s))$ at every point $z(s)$. Then we write $G \in$ $C_{\theta} \Leftrightarrow \partial G \in C_{\theta}$.

According to the "three-point" criterion [13, p.100], every piecewise smooth curve (without any cusps) is quasiconformal. Moreover, according to [23], we have the following:

Corollary 3. If $G \in C_{\theta}$, then $\partial G$ is $(1+\varepsilon)$-quasiconformal for arbitrary small $\varepsilon>0$.

Now we give the definitions of regions with a piecewise smooth curve, which we present our main result and some notation that will be used later in the text.

Definition 4. [5] We say that a Jordan region $G \in C_{\theta}\left(\lambda_{1}, \ldots, \lambda_{l}\right), 0<\lambda_{j} \leq 2, j=\overline{1, l}$, if $L=\partial G$ consists of the union of finite smooth $\operatorname{arcs}\left\{L_{j}\right\}_{j=1}^{l}$, such that they have exterior (with respect to $\bar{G}$ ) angles $\lambda_{j} \pi, 0<\lambda_{j} \leq 2$, at the corner points $\left\{z_{j}\right\}_{j=1}^{l} \in L$, where two arcs meet.

Without loss of generality, we assume that these points on the curve $L=\partial G$ are located in the positive direction such that, $G$ has exterior $\lambda_{j} \pi, 0<\lambda_{j}<2, j=\overline{0, l_{1}}$, angle at the points $\left\{z_{j}\right\}_{j=1}^{l_{1}}, l_{1} \leq l$, and interior zero angle (i.e. $\lambda_{j}=2-$ interior cusps) at the points $\left\{z_{j}\right\}_{j=l_{1}+1}^{l}$.

It is clear from Definition 4 , the each region $G \in C_{\theta}\left(\lambda_{1}, \ldots, \lambda_{l}\right), 0<\lambda_{j} \leq 2, j=\overline{1, l}$, may have exterior nonzero $\lambda_{j} \pi$, $0<$ $\lambda_{j}<2$, angles at the points $\left\{z_{j}\right\}_{j=1}^{l_{1}} \in L$, and interior zero angles $\left(\lambda_{j}=2\right)$ at the the points $\left\{z_{j}\right\}_{j=l_{1}+1}^{l} \in L$. If $l_{1}=l=0$, then the region $G$ doesn't have such angles, and in this case we will write: $G \in C_{\theta}$; if $l_{1}=l \geq 1$, then $G$ has only $\lambda_{i} \pi, 0<\lambda_{i}<2$, $i=\overline{1}_{1}$, exterior nonzero angles, and in this case we will write: $G \in C_{\theta}\left(\lambda_{i}\right)$; if $l_{1}=0$ and $l \geq 1$, then $G$ has only interior zero angles, and in this case we will write: $G \in C_{\theta}(2)$.

Throughout this work, we will assume that the points $\left\{z_{j}\right\}_{j=1}^{l} \in L$ defined in (1) and Definition 4 are identical and $w_{j}:=\Phi\left(z_{j}\right)$. For simplicity of exposition and in order to avoid cumbersome calculations, without loss of generality, we will take $l_{1}=1, l=$ 2. Then, after this assumption, in the future we will have region $G \in C_{\theta}\left(\lambda_{1}, 2\right), 0<\lambda_{1}<2$, such that at the point $z_{1} \in L$ region $G$ have exterior nonzero $\lambda_{1} \pi, \quad 0<\lambda_{1}<2$, and at the point $z_{2} \in L$ - interior zero angle. Note that, the notation " $G \in C_{\theta}\left(\lambda_{1}, \lambda_{2}\right)$, $0<\lambda_{1}, \lambda_{2}<2$ " means that the region $G$ has two exterior nonzero $\lambda_{j} \pi, 0<\lambda_{j}<2, j=1,2$, angles at the point $z_{j} \in L$.

For $0<\delta_{j}<\delta_{0}:=\frac{1}{4} \min \left\{\left|z_{1}-z_{2}\right|: j=1,2\right\}, \delta:=\min _{1 \leq j \leq 2} \delta_{j}$, let

$$
\begin{aligned}
& \Omega\left(z_{j}, \delta_{j}\right):=\Omega \cap\left\{z:\left|z-z_{j}\right| \leq \delta_{j}\right\} \\
& \Omega(\delta):=\bigcup_{j=1}^{2} \Omega\left(z_{j}, \delta\right), \widehat{\Omega}:=\Omega \backslash \Omega(\delta) .
\end{aligned}
$$

In this work, we study problem of (8) type in regions with piecewise smooth boundary without exterior cusps and generalized Jacobi weight function $h(z)$, as defined in (1).

Now, we start to formulate the new results.

Theorem 5. Let $p>1 ; G \in C_{\theta}\left(\lambda_{1}, 2\right)$, for some $0<\lambda_{1}<2 ; h(z)$ be defined as in (1). Then, for any $P_{n} \in \wp_{n}, n \in \mathbb{N}, \gamma_{j}>$ -2 and arbitrary small $\varepsilon>0$ 


$$
\left|P_{n}^{\prime}(z)\right| \leq c_{1}\left[\frac{|\Phi(z)|^{n+1}}{d\left(z, L_{R_{1}}\right)} G_{n, 1}(z)+\frac{|\Phi(z)|^{2(n+1)}}{d^{2 / p}\left(z, L_{1+1 / n}\right)} B_{n, 1}(z) E_{n, 1}\right]\left\|P_{n}\right\|_{p}
$$

holds, where $c_{1}=c_{1}\left(G, \gamma_{i}, p, \varepsilon\right)>0$;

$$
\begin{aligned}
& G_{n, 1}(z):=\left\{\begin{array}{ccc}
n^{\frac{\gamma_{1}+2}{p}} \tilde{\lambda}_{1}, & \gamma_{1} \geq \frac{2}{\tilde{\lambda}_{1}}\left(\gamma_{2}+2\right)-2, \gamma_{2} \geq \frac{\tilde{\lambda}_{1}}{2 \lambda_{1}}-2, & z \in \Omega(\delta), \\
n^{\frac{\gamma_{2}+2}{p} 2}, & \frac{1}{\lambda_{1}}-2 \leq \gamma_{1}<\frac{2}{\tilde{\lambda}_{1}}\left(\gamma_{2}+2\right)-2, \gamma_{2} \geq \frac{\widetilde{\lambda}_{1}}{2 \lambda_{1}}-2, & z \in \Omega(\delta), \\
n^{\frac{\gamma_{2}+2}{p} 2}, & \gamma_{1}<\frac{1}{\lambda_{1}}-2, \gamma_{2} \geq \frac{\tilde{\lambda}_{1}}{2 \lambda_{1}}-2, & z \in \Omega(\delta), \\
1, & \gamma_{1}<\frac{1}{\lambda_{1}}-2, \gamma_{2}<-\frac{3}{2}, & z \in \Omega(\delta), \\
n^{\frac{2}{p}+\varepsilon}, & \text { for all } \lambda_{1}, \gamma_{1}, \gamma_{2}, & z \in \widehat{\Omega}(\delta),
\end{array}\right. \\
& E_{n ; 1}:=\left\{\begin{array}{ll}
n^{\frac{\tilde{\gamma} \cdot \hat{\lambda}}{p}}, & \text { if } \tilde{\gamma} \cdot \hat{\lambda} \geq 1, \\
n^{\frac{1}{p}}, & \text { if } \tilde{\gamma} \cdot \hat{\lambda}<1,
\end{array} \quad B_{n, 1}(z):=\left\{\begin{array}{ll}
n^{\hat{\lambda}}, & z \in \Omega(\delta), \\
n^{1+\varepsilon}, & z \in \widehat{\Omega}(\delta),
\end{array} ;\right.\right. \\
& \hat{\lambda}:=\left\{\begin{array}{ll}
\max \{1 ; \lambda\}+\varepsilon, & \text { if } 0<\lambda<2, \\
2, & \text { if } \lambda=2,
\end{array} ; \quad \tilde{\gamma}:= \begin{cases}\tilde{\gamma}_{1}, & \text { if } 0<\lambda<2, \\
\tilde{\gamma}_{2}, & \text { if } \lambda=2,\end{cases} \right. \\
& \tilde{\gamma}_{i}:=\max \left\{0 ; \gamma_{i}\right\}, \quad i=1,2 ; \quad \tilde{\lambda}_{1}:=\max \left\{1 ; \lambda_{1}\right\}+\varepsilon .
\end{aligned}
$$

Theorem 6. Let $p>1 ; G \in C_{\theta}\left(\lambda_{1}, \lambda_{2}\right)$, for some $0<\lambda_{j}<2, j=1,2 ; h(z)$ be defined as in (1). Then, for any $P_{n} \in \wp_{n}, n \in$ $\mathbb{N}, \gamma_{j}>-2$ and arbitrary small $\varepsilon>0$

$$
\left|P_{n}^{\prime}(z)\right| \leq c_{2}\left\|P_{n}\right\|_{p}\left[\frac{|\Phi(z)|^{n+1}}{d\left(z, L_{R_{1}}\right)} G_{n, 2}(z)+\frac{|\Phi(z)|^{2(n+1)}}{d^{2 / p}\left(z, L_{1+1 / n}\right)} B_{n, 2}(z) E_{n, 2}\right]
$$

holds, where $c_{2}=c_{2}\left(G, \gamma_{i}, p, \varepsilon\right)>0$;

$$
\begin{aligned}
& G_{n, 2}(z):=\left\{\begin{array}{ccc}
n^{\frac{\gamma_{1}+2}{p} \tilde{\lambda}_{1},} & \gamma_{1} \geq \frac{1}{\lambda_{1}}-2, \gamma_{2}<\frac{1}{\lambda_{2}}-2, & z \in \Omega(\delta), \\
n^{\frac{\gamma_{2}+2}{p} \tilde{\lambda}_{2},} & \gamma_{1}<\frac{1}{\lambda_{1}}-2, \gamma_{2} \geq \frac{1}{\lambda_{2}}-2, & z \in \Omega(\delta), \\
1, & \gamma_{1}<\frac{1}{\lambda_{1}}-2, \gamma_{2}<\frac{1}{\lambda_{2}}-2, & z \in \Omega(\delta), \\
n^{\frac{\gamma_{1}+2}{p} \tilde{\lambda}_{1},} & \gamma_{1} \geq \frac{\lambda_{2}}{\lambda_{1}}\left(\gamma_{2}+2\right)-2, \gamma_{2} \geq \frac{1}{\lambda_{2}}-2, & z \in \Omega(\delta), \\
n^{\frac{\gamma_{2}+2}{p}} \tilde{\lambda}_{2}, & \frac{1}{\lambda_{1}}-2 \leq \gamma_{1}<\frac{\lambda_{2}}{\lambda_{1}}\left(\gamma_{2}+2\right)-2, \gamma_{2} \geq \frac{1}{\lambda_{2}}-2, & z \in \Omega(\delta), \\
n^{\frac{2}{p}+\varepsilon}, & \text { for all } \lambda_{1}, \gamma_{1}, & z \in \widehat{\Omega}(\delta),
\end{array}\right. \\
& E_{n, 2}:=\left\{\begin{array}{ll}
n^{\frac{\tilde{\gamma} \cdot \tilde{\lambda}}{p}}, & \text { if } \tilde{\gamma} \cdot \tilde{\lambda} \geq 1, \\
n^{\frac{1}{p}}, & \text { if } \tilde{\gamma} \cdot \tilde{\lambda}<1,
\end{array} ; B_{n, 2}(z):=\left\{\begin{array}{ll}
n^{\tilde{\lambda}}, & z \in \Omega(\delta), \\
n^{1+\varepsilon}, & z \in \widehat{\Omega}(\delta),
\end{array} ;\right.\right. \\
& \hat{\lambda}:=\left\{\begin{array}{ll}
\max \{1 ; \lambda\}+\varepsilon, & \text { if } 0<\lambda<2, \\
2, & \text { if } \lambda=2,
\end{array} ; \quad \tilde{\gamma}:= \begin{cases}\tilde{\gamma}_{1}, & \text { if } 0<\lambda<2, \\
\tilde{\gamma}_{2}, & \text { if } \lambda=2,\end{cases} \right. \\
& \tilde{\gamma}_{i}:=\max \left\{0 ; \gamma_{i}\right\}, \quad \tilde{\lambda}_{i}:=\max \left\{1 ; \lambda_{i}\right\}+\varepsilon, i=1,2 \text {. }
\end{aligned}
$$

Analogously, we also can give a theorem for the regions such as $G \in C_{\theta}(2,2)$. 


\section{Some auxiliary results}

Lemma 1. [1] Let $L$ be a $K$-quasiconformal curve, $z_{1} \in L, z_{2}, z_{3} \in \Omega \cap\left\{z:\left|z-z_{1}\right| \prec d\left(z_{1}, L_{R_{0}}\right)\right\} ; w_{j}=\Phi\left(z_{j}\right), \quad\left(z_{2}, z_{3} \in\right.$ $\left.G \cap\left\{z:\left|z-z_{1}\right| \prec d\left(z_{1}, L_{R_{0}}\right)\right\} ; w_{j}=\varphi\left(z_{j}\right)\right), j=1,2,3$. Then

a) The statements $\left|\mathrm{z}_{1}-\mathrm{z}_{2}\right|<\left|\mathrm{z}_{1}-\mathrm{z}_{3}\right|$ and $\left|\mathrm{w}_{1}-\mathrm{w}_{2}\right|<\left|\mathrm{w}_{1}-\mathrm{w}_{3}\right|$ are equivalent.

So are $\left|z_{1}-z_{2}\right|=\left|z_{1}-z_{3}\right|$ and $\left|w_{1}-w_{2}\right|=\left|w_{1}-w_{3}\right|$.

b) If $\left|z_{1}-z_{2}\right|<\left|z_{1}-z_{3}\right|$, then

$$
\left|\frac{w_{1}-w_{3}}{w_{1}-w_{2}}\right|^{K^{2}}<\left|\frac{z_{1}-z_{3}}{z_{1}-z_{2}}\right|<\left|\frac{w_{1}-w_{3}}{w_{1}-w_{2}}\right|^{K^{-2}}
$$

where $\varepsilon<1, c>1, R_{0}>1$ are constants, depending on $G$.

Corollary 7. Under the assumptions of Lemma 1 , if $z_{3} \in L_{R_{0}}$, then

$$
\left|w_{1}-w_{2}\right|^{K^{2}}<\left|z_{1}-z_{2}\right|<\left|w_{1}-w_{2}\right|^{K^{-2}} .
$$

Corollary 8. If $L \in C_{\theta}$, then

$$
\left|w_{1}-w_{2}\right|^{1+\varepsilon} \prec\left|z_{1}-z_{2}\right| \prec\left|w_{1}-w_{2}\right|^{1-\varepsilon}
$$

for all $\varepsilon>0$.

The following lemma is a consequence of the results given in [18], [21], [27] and of estimate for the $\left|\Psi^{\prime}\right|$ (see, for example, $[14$, Th.2.8]):

$$
\left|\Psi^{\prime}(\tau)\right|=\frac{d(\Psi(\tau), L)}{|\tau|-1} .
$$

Let $w_{j}:=\Phi\left(z_{j}\right), \varphi_{j}:=\arg w_{j}$. Without loss of generality, we will assume that $\varphi_{l}<2 \pi$. Additionally to the notations (9), for $\eta_{j}=\min _{t \in \partial \Phi\left(\Omega\left(z_{j}, \delta_{j}\right)\right)}\left|t-w_{j}\right|>0$ and $\eta:=\min \left\{\eta_{j}, j=\overline{1, l}\right\}$ let us set: $\Delta_{j}\left(\eta_{j}\right):=\left\{t:\left|t-w_{j}\right| \leq \eta_{j}\right\} \subset \Phi\left(\Omega\left(z_{j}, \delta_{j}\right)\right), \Delta(\eta):=$ $\bigcup_{j=1}^{l} \Delta_{j}(\eta), \widehat{\Delta}_{j}=\Delta \backslash \Delta\left(\eta_{j}\right) ; \widehat{\Delta}(\eta):=\Delta \backslash \Delta(\eta) ; \quad \Delta_{1}^{\prime}:=\Delta_{1}^{\prime}(1), \Delta_{1}^{\prime}(\rho):=\left\{t=R \cdot e^{i \theta}: R \geq \rho>1, \frac{\varphi_{0}+\varphi_{1}}{2} \leq \theta<\frac{\varphi_{1}+\varphi_{2}}{2}\right\}, \Delta_{j}^{\prime}:=$ $\Delta_{j}^{\prime}(1), \Delta_{j}^{\prime}(\rho):=\left\{t=R \cdot e^{i \theta}: R \geq \rho>1, \frac{\varphi_{j-1}+\varphi_{j}}{2} \leq \theta<\frac{\varphi_{j}+\varphi_{0}}{2}\right\}, j=2,3 \ldots, l$, where $\varphi_{0}=2 \pi-\varphi_{l} ; \Omega_{j}:=\Psi\left(\Delta_{j}^{\prime}\right), L_{R_{1}}^{j}:=$ $L_{R_{1}} \cap \Omega_{j}$. Clearly, $\Omega=\bigcup_{j=1}^{l} \Omega_{j}$.

The following lemma is a consequence of the results given in [27] and [18].

Lemma 2. Let $G \in C_{\theta}\left(\lambda, \ldots, \lambda_{l}\right), 0<\lambda_{j}<2, j=1,2, \ldots, l$,. Then

i) for any $w \in \Delta_{j},\left|w-w_{j}\right|^{\lambda_{j}+\varepsilon} \prec\left|\Psi(w)-\Psi\left(w_{j}\right)\right| \prec\left|w-w_{j}\right|^{\lambda_{j}-\varepsilon},\left|w-w_{j}\right|^{\lambda_{j}-1+\varepsilon}<\left|\Psi^{\prime}(w)\right| \prec\left|w-w_{j}\right|^{\lambda_{j}-1-\varepsilon}$,

ii) for any $w \in \bar{\Delta} \backslash \Delta_{j},(|w|-1)^{1+\varepsilon} \prec d(\Psi(w), L)\left|<(|w|-1)^{1-\varepsilon},(|w|-1)^{\varepsilon}<\right| \Psi^{\prime}(w) \mid \prec(|w|-1)^{-\varepsilon}$.

Let $\left\{z_{j}\right\}_{j=1}^{l}$ be a fixed system of distinct points on curve $L$ ordered in the positive direction and the weight function $h(z)$ be defined as in (1).

Lemma 3. [6] Let $L$ is a $K$-quasiconformal curve; $R=1+\frac{c}{n}$. Then, for any fixed $\varepsilon \in(0,1)$ there exist a level curve $L_{1+\varepsilon(R-1)}$ such that the following holds for any polynomial $P_{n}(z) \in \wp_{n}, n \in \mathbb{N}$ :

$$
\left\|P_{n}\right\|_{\mathcal{L}_{p}\left(\frac{h}{\left|\Phi^{\prime}\right|} L_{1+\varepsilon(R-1)}\right)} \prec n^{\frac{1}{p}}\left\|P_{n}\right\|_{p}, p>0 .
$$

Lemma 4. [6] Let $L$ be a $K$-quasiconformal curve; $h(z)$ be defined as in (1). Then, for arbitrary $P_{n}(z) \in \wp_{n}$, any $R>1$ and $n=1,2, \ldots$, we have 


$$
\left\|P_{n}\right\|_{A_{p}\left(h, G_{R}\right)} \prec \tilde{R}^{n+\frac{1}{p}}\left\|P_{n}\right\|_{A_{p}(h, G)}, p>0,
$$

where $\widetilde{R}=1+c(R-1)$ and $c$ is independent from $n$ and $R$.

Lemma 5. Let $G \in C_{\theta}\left(\lambda_{1}, \ldots, \lambda_{l}\right), 0<\lambda_{j} \leq 2, j=\overline{1, l}$. Then, for arbitrary $P_{n}(z) \in \wp_{n}$ and any $p>0$, we have:

$$
\left\|P_{n}\right\|_{A_{p}\left(h, G_{1+c / n}\right)} \prec\left\|P_{n}\right\|_{A_{p}(h, G)} \text {. }
$$

\section{Proof of Theorems}

\subsection{Proof of Theorems 5 and 6.}

Proof. We will prove both theorems simultaneously. Suppose that $G \in C_{\theta}(\lambda ; 2)\left(C_{\theta}\left(\lambda_{1} ; \lambda_{2}\right)\right)$ for some $0<\lambda<2 ; h(z)$ be defined as in (1). For $z \in \Omega$, we define:

Then

$$
T_{n}(z):=\frac{P_{n}(z)}{\Phi^{n+1}(z)} .
$$

$$
T_{n}^{\prime}(z)=\frac{P_{n}^{\prime}(z)}{\Phi^{n+1}(z)}+P_{n}(z)\left(\frac{1}{\Phi^{n+1}(z)}\right)^{\prime}, z \in \Omega
$$

For any $R>1$ and $R_{1}:=1+\frac{R-1}{2}$, Cauchy integral representation for the region $\Omega_{R_{1}}$ gives

$$
\begin{aligned}
T_{n}^{\prime}(z) & =-\frac{1}{2 \pi i} \int_{L_{R_{1}}} T_{n}(\zeta) \frac{d \zeta}{(\zeta-z)^{2}} \\
& =-\frac{1}{2 \pi i} \int_{L_{R_{1}}} \frac{P_{n}(\zeta)}{\Phi^{n+1}(\zeta)} \frac{d \zeta}{(\zeta-z)^{2}}, \quad z \in \Omega_{R_{1}},
\end{aligned}
$$

and

$$
\left(\frac{1}{\Phi^{n+1}(z)}\right)^{\prime}=-\frac{1}{2 \pi i} \int_{L_{R_{1}}} \frac{1}{\Phi^{n+1}(\zeta)} \frac{d \zeta}{(\zeta-z)^{2}}, z \in \Omega_{R_{1}} .
$$

Then, from (15), we get

$$
\begin{gathered}
P_{n}^{\prime}(z)=\Phi^{n+1}(z)\left[T_{n}^{\prime}(z)-P_{n}(z)\left(\frac{1}{\Phi^{n+1}(z)}\right)^{\prime}\right] \\
=\Phi^{n+1}(z)\left[-\frac{1}{2 \pi i} \int_{L_{R_{1}}} \frac{P_{n}(\zeta)}{\Phi^{n+1}(\zeta)} \frac{d \zeta}{(\zeta-z)^{2}}+\frac{P_{n}(z)}{2 \pi i} \int_{L_{R_{1}}} \frac{1}{\Phi^{n+1}(\zeta)} \frac{d \zeta}{(\zeta-z)^{2}}\right], \quad z \in \Omega_{R_{1}} .
\end{gathered}
$$

Therefore,$$
\left|P_{n}^{\prime}(z)\right| \leq \frac{\left|\Phi^{n+1}(z)\right|}{2 \pi}\left[\int_{L_{R_{1}}}\left|\frac{P_{n}(\zeta)}{\Phi^{n+1}(\zeta)}\right| \frac{|d \zeta|}{|\zeta-z|^{2}}+\left|P_{n}(z)\right| \int_{L_{R_{1}}}\left|\frac{1}{\Phi^{n+1}(\zeta)}\right| \frac{|d \zeta|}{|\zeta-z|^{2}}\right] .
$$

Since $|\Phi(\zeta)|>1$, for $\zeta \in L_{R_{1}}$, then, we have:

$$
\begin{aligned}
\left|P_{n}^{\prime}(z)\right| & <\frac{|\Phi(z)|^{n+1}}{2 \pi}\left[\int_{L_{R_{1}}}\left|P_{n}(\zeta)\right| \frac{|d \zeta|}{|\zeta-z|^{2}}+\left|P_{n}(z)\right| \int_{L_{R_{1}}} \frac{|d \zeta|}{|\zeta-z|^{2}}\right] \\
& \leq \frac{|\Phi(z)|^{n+1}}{2 \pi}\left[\frac{1}{d\left(z, L_{R_{1}}\right)} \int_{L_{R_{1}}}\left|P_{n}(\zeta)\right| \frac{|d \zeta|}{|\zeta-z|}+\left|P_{n}(z)\right| \int_{L_{R_{1}}} \frac{|d \zeta|}{|\zeta-z|^{2}}\right] .
\end{aligned}
$$

Denote by

$$
A_{n}(z):=\int_{L_{R_{1}}}\left|P_{n}(\zeta)\right| \frac{|d \zeta|}{|\zeta-z|} ; B_{n}(z):=\int_{L_{R_{1}}} \frac{|d \zeta|}{|\zeta-z|^{2}}
$$


and will be estimate these integrals separately.

To estimate $A_{n}(z)$, first of all replacing the variable $\tau=\Phi(\zeta)$ and multiplying the numerator and denominator of the integrant by $\prod_{j=1}^{2}\left|\Psi(\tau)-\Psi\left(w_{j}\right)\right|^{\frac{\gamma_{j}}{p}}\left|\Psi^{\prime}(\tau)\right|^{\frac{2}{p}}$ and applying the Hölder inequality, we obtain:

$$
\begin{gathered}
A_{n}(z)=\int_{L_{R_{1}}}\left|P_{n}(\zeta)\right| \frac{|d \zeta|}{|\zeta-z|} \\
=\sum_{i=1}^{3} \int_{F_{R_{1}}^{i}} \frac{\left.\prod_{j=1}^{2}\left|\Psi(\tau)-\Psi\left(w_{j}\right)\right|^{\frac{\gamma_{j}}{p}}\left|P_{n}(\Psi(\tau))\left(\Psi^{\prime}(\tau)\right)^{\frac{2}{p}}\right| \Psi^{\prime}(\tau)\right|^{1-\frac{2}{p}}}{\prod_{j=1}^{2}\left|\Psi(\tau)-\Psi\left(w_{j}\right)\right|^{\frac{\gamma_{j}}{p}}|\Psi(\tau)-\Psi(w)|}|d \tau| \\
\leq \sum_{i=1}^{3}\left(\int_{F_{R_{1}}^{i}} \prod_{j=1}^{2}\left|\Psi(\tau)-\Psi\left(w_{j}\right)\right|^{\gamma_{j}}\left|P_{n}(\Psi(\tau))\right|^{p}\left|\Psi^{\prime}(\tau)\right|^{2}|d \tau|\right)^{\frac{1}{p}} \\
\times\left(\int_{F_{R_{1}}^{i}}\left(\frac{\left|\Psi^{\prime}(\tau)\right|^{1-\frac{2}{p}}}{\prod_{j=1}^{2}\left|\Psi(\tau)-\Psi\left(w_{j}\right)\right|^{\frac{\gamma_{j}}{p}}|\Psi(\tau)-\Psi(w)|}\right)^{q}|d \tau|\right)^{\frac{1}{q}} \\
=: \sum_{i=1}^{3} A_{n}^{i}(z),
\end{gathered}
$$

where $F_{R_{1}}^{j}:=\Phi\left(L_{R_{1}}^{1}\right)=\Delta_{j}^{\prime} \cap\left\{\tau:|\tau|=R_{1}\right\}, j=1,2 ; F_{R_{1}}^{3}:=\Phi\left(L_{R_{1}}^{1}\right) \backslash\left(F_{R_{1}}^{1} \cup F_{R_{1}}^{21}\right)$ and

$$
\begin{gathered}
A_{n}^{i}(z):=\left(\int_{F_{R_{1}}^{i}}\left|f_{n, p}(\tau)\right|^{p}|d \tau|\right)^{\frac{1}{p}}\left(\int_{F_{R_{1}}^{i}} \frac{\left|\Psi^{\prime}(\tau)\right|^{2-q}}{\prod_{j=1}^{2}\left|\Psi(\tau)-\Psi\left(w_{j}\right)\right|^{\gamma_{j}(q-1)}|\Psi(\tau)-\Psi(w)|^{q}}|d \tau|\right)^{\frac{1}{q}} \\
=: J_{n, 1}^{i} \cdot J_{n, 2}^{i}(z), \\
f_{n, p}(\tau):=h^{\frac{1}{p}}(\Psi(\tau)) P_{n}(\Psi(\tau))\left(\Psi^{\prime}(\tau)\right)^{\frac{2}{p}},|\tau|=R_{1} .
\end{gathered}
$$

Applying to Lemmas 3, 4 and 5, we get:

$$
J_{n, 1}^{i} \prec n^{\frac{1}{p}}\left\|P_{n}\right\|_{p}, \quad i=1,2,3
$$

For the estimation of the integral $J_{n, 2}^{i}(z)$, for $i=1,2,3$, and $j=1,2$, we set:

$$
\begin{aligned}
& E_{R_{1}}^{11}\left(w_{j}\right)=\left\{\tau: \tau \in F_{R_{1}}^{j},\left|\tau-w_{j}\right|<c_{j}\left(R_{1}-1\right)\right\}, \\
& E_{R_{1}}^{12}\left(w_{j}\right):=\left\{\tau: \tau \in F_{R_{1}}^{j}, c_{j}\left(R_{1}-1\right) \leq\left|\tau-w_{j}\right|<\eta\right\}, \\
& E_{R_{1}}^{13}\left(w_{j}\right):=\left\{\tau: \tau \in \Phi\left(L_{R_{1}}^{j}\right),\left|\tau-w_{j}\right| \geq \eta\right\},
\end{aligned}
$$

where $0<c_{j}<\eta$ is chosen so that $\left\{\tau:\left|\tau-w_{j}\right|<c_{j}\left(R_{1}-1\right)\right\} \cap \Delta \neq \varnothing$ and $\Phi\left(L_{R_{1}}^{j}\right)=\bigcup_{k=1}^{3} E_{R_{1}}^{1 k}\left(w_{j}\right)$. Taking into consideration these notations, (19) can be written as:

and, consequently,

$$
\begin{aligned}
\sum_{i=1}^{3} J_{n, 2}^{i}(z) & =: J_{2}(z)=\sum_{i=1}^{3} \sum_{j=1}^{2} J_{2}\left(E_{R_{1}}^{1 i}\left(w_{j}\right), z\right) \\
& =: \sum_{i=1}^{3} \sum_{j=1}^{2} J_{2, j}^{i}(z)
\end{aligned}
$$

$$
A_{n}(z) \prec n^{\frac{1}{p}}\left\|P_{n}\right\|_{p} \cdot \sum_{j=1}^{2} \sum_{i=1}^{3} J_{2, j}^{i}(z)=: \sum_{j=1}^{2} \sum_{i=1}^{3} A_{n, i}^{j}(z),
$$

where

$$
A_{n, i}^{j}(z):=n^{\frac{1}{p}}\left\|P_{n}\right\|_{p} \cdot J_{2, j}^{i}(z), i=1,2,3 ; j=1,2 .
$$




$$
\begin{aligned}
\left(J_{2, j}^{i}(z)\right)^{q}: & =\int_{E_{R_{1}}^{1 i}\left(w_{j}\right)} \frac{\left|\Psi^{\prime}(\tau)\right|^{2-q}|d \tau|}{\prod_{j=1}^{2}\left|\Psi(\tau)-\Psi\left(w_{j}\right)\right|^{\gamma_{j}(q-1)}|\Psi(\tau)-\Psi(w)|^{q}} \\
& =\sum_{j=1}^{2} \int_{E_{R_{1}}^{1 i}\left(w_{j}\right)} \frac{\left|\Psi^{\prime}(\tau)\right|^{2-q}|d \tau|}{\left|\Psi(\tau)-\Psi\left(w_{j}\right)\right|^{\gamma_{j}(q-1)}|\Psi(\tau)-\Psi(w)|^{q}}, i=1,2,3,
\end{aligned}
$$

since the points $w_{1}$ and $w_{2}$ are isolated.

Therefore, we need to estimate the quantity (21). In case of $j=1$, for any $p>1,0<\lambda_{1}<2, \gamma>-2$, and for all sufficiently small $\varepsilon>0$, in [12] is proved following estimate:

$$
\sum_{i=1}^{3} A_{n, i}^{1}(z) \prec\left\|P_{n}\right\|_{p} \cdot\left\{\begin{array}{cl}
n^{\frac{\gamma_{1}+2}{p} \tilde{\lambda}_{1},} \begin{array}{l}
0<\lambda_{1}<2, \\
\gamma_{1} \geq \frac{1}{\lambda_{1}}-2,
\end{array} \quad z \in \Omega(\delta), \\
n^{\frac{2}{p}+\varepsilon}, & \begin{array}{l}
\text { for all } \lambda_{1}, \gamma_{1}, \quad \\
\text { otherwise, }
\end{array}
\end{array}\right.
$$

where $\tilde{\lambda}_{1}:=\max \left\{1 ; \lambda_{1}\right\}+\varepsilon$.

Similarly to the case $j=1$, for the case $j=2$, we obtain:

$$
\sum_{i=1}^{3} A_{n, i}^{2}(z) \prec\left\|P_{n}\right\|_{p} \cdot\left\{\begin{array}{cll}
n^{\frac{\gamma_{2}+2}{p} 2+\varepsilon}, & \gamma_{2} \geq-\frac{3}{2}, & z \in \Omega(\delta), \\
n^{\frac{2}{p}+\varepsilon}, & \gamma_{1}>-2, & z \in \widehat{\Omega}(\delta), \\
1, & \text { otherwise }
\end{array}\right.
$$

Combining (23) and (24), for the region $G \in C_{\theta}\left(\lambda_{1}, 2\right)$, any $p>1, \gamma_{1}>-2,0<\lambda_{1}<2$, and for all sufficiently small $\varepsilon>0$, we obtain:

$$
\begin{aligned}
& A_{n}(z)=\sum_{k=1}^{3} A_{n, k}^{1} \prec\left\|P_{n}\right\|_{p} \times \\
& \times\left\{\begin{array}{cll}
n^{\frac{\gamma_{1}+2}{p} \tilde{\lambda}_{1},} & \gamma_{1} \geq \frac{1}{\lambda_{1}}-2, & z \in \Omega(\delta), \\
n^{\frac{2}{p}+\varepsilon}, & \text { for all } \lambda_{1}, \gamma_{1}, & z \in \widehat{\Omega}(\delta), \\
1, & \text { otherwise }
\end{array}+\left\{\begin{array}{cll}
n^{\frac{\gamma_{2}+2}{p} 2+\varepsilon,} & \gamma_{2} \geq-\frac{3}{2}, & z \in \Omega(\delta), \\
n^{\frac{2}{p}+\varepsilon}, & \begin{array}{l}
\gamma_{1}>-2, \\
1,
\end{array} \quad \text { otherwise } & z \in \widehat{\Omega}(\delta),
\end{array}\right.\right. \\
& \prec\left\|P_{n}\right\|_{p} \cdot\left\{\begin{array}{ccc}
n^{\frac{\gamma_{1}+2}{p} \tilde{\lambda}_{1}}, & \gamma_{1} \geq \frac{2}{\bar{\lambda}_{1}}\left(\gamma_{2}+2\right)-2, \gamma_{2} \geq \frac{\tilde{\lambda}_{1}}{2 \lambda_{1}}-2, & z \in \Omega(\delta), \\
n^{\frac{\gamma_{2}+2}{p} 2}, & \frac{1}{\lambda_{1}}-2 \leq \gamma_{1}<\frac{2}{\tilde{\lambda}_{1}}\left(\gamma_{2}+2\right)-2, \gamma_{2} \geq \frac{\tilde{\lambda}_{1}}{2 \lambda_{1}}-2, & z \in \Omega(\delta), \\
n^{\frac{\gamma_{2}+2}{p} 2}, & \gamma_{1}<\frac{1}{\lambda_{1}}-2, \gamma_{2} \geq \frac{\tilde{\lambda}_{1}}{2 \lambda_{1}}-2, & z \in \Omega(\delta), \\
1, & \gamma_{1}<\frac{1}{\lambda_{1}}-2, \gamma_{2}<-\frac{3}{2}, & z \in \Omega(\delta), \\
n^{\frac{2}{p}+\varepsilon}, & \text { for all } \lambda_{1}, \gamma_{1}, \gamma_{2}, & z \in \widehat{\Omega}(\delta),
\end{array}\right. \\
& =:\left\|P_{n}\right\|_{p} \cdot G_{n, 1}(z)
\end{aligned}
$$

If the angle at point $z_{2}$ is equals $\lambda_{2} \pi$ such that $0<\lambda_{1}, \lambda_{2}<2$, , then, analogously to (25), for the region $G \in C_{\theta}\left(\lambda_{1}, \lambda_{2}\right)$ all $0<$ $\lambda_{1}, \lambda_{2}<2$,we have:

$$
A_{n}(z)=\sum_{k=1}^{3} A_{n, k}^{1} \prec\left\|P_{n}\right\|_{p} \times
$$




$$
\begin{aligned}
& \left\{\begin{array}{cll}
n^{\frac{\gamma_{1}+2}{p}} \widetilde{\lambda}_{1}, & \gamma_{1} \geq \frac{1}{\lambda_{1}}-2, & z \in \Omega(\delta), \\
n^{\frac{2}{p}+\varepsilon}, & \begin{array}{l}
\text { for all } \lambda_{1}, \gamma_{1}, \\
\text { otherwise }
\end{array}, \quad z \in \widehat{\Omega}(\delta),
\end{array}+\left\{\begin{array}{cll}
n^{\frac{\gamma_{2}+2}{p} \widetilde{\lambda}_{2},} & \gamma_{2} \geq \frac{1}{\lambda_{2}}-2, & z \in \Omega(\delta), \\
n^{\frac{2}{p}+\varepsilon}, & \text { for all } \lambda_{2}, \gamma_{2}, & z \in \widehat{\Omega}(\delta), \\
1, & \text { otherwise }
\end{array}\right.\right. \\
& \prec\left\|P_{n}\right\|_{p} \times\left\{\begin{array}{ccc}
n \frac{\gamma_{1}+2}{p} \tilde{\lambda}_{1}, & \gamma_{1} \geq \frac{1}{\lambda_{1}}-2, \gamma_{2}<\frac{1}{\lambda_{2}}-2, & z \in \Omega(\delta), \\
n \frac{\gamma_{2}+2}{p} \tilde{\lambda}_{2}, & \gamma_{1}<\frac{1}{\lambda_{1}}-2, \gamma_{2} \geq \frac{1}{\lambda_{2}}-2, & z \in \Omega(\delta), \\
1, & \gamma_{1}<\frac{1}{\lambda_{1}}-2, \gamma_{2}<\frac{1}{\lambda_{2}}-2, & z \in \Omega(\delta), \\
n^{\frac{\gamma_{1}+2}{p} \tilde{\lambda}_{1},} & \gamma_{1} \geq \frac{\lambda_{2}}{\lambda_{1}}\left(\gamma_{2}+2\right)-2, \gamma_{2} \geq \frac{1}{\lambda_{2}}-2, & z \in \Omega(\delta), \\
n^{\frac{\gamma_{2}+2}{p} \tilde{\lambda}_{2},} \frac{1}{\lambda_{1}}-2 \leq \gamma_{1}<\frac{\lambda_{2}}{\lambda_{1}}\left(\gamma_{2}+2\right)-2, \gamma_{2} \geq \frac{1}{\lambda_{2}}-2, & \text { for all } \lambda_{1}, \gamma_{1}, & z \in \widehat{\Omega}(\delta),
\end{array}\right. \\
& =:\left\|P_{n}\right\|_{p} \times G_{n, 2}(z) \text {. }
\end{aligned}
$$

Now, let us estimate $B_{n}(z)$. Let $G \in C_{\theta}\left(\lambda_{1}, 2\right)$. By replacing the variable $\tau=\Phi(\zeta)$ and according to (10) and Lemma 2 , we obtain:

$$
\begin{aligned}
& B_{n}(z)=\int_{L_{R_{1}}} \frac{|d \zeta|}{|\zeta-z|^{2}}=\int_{|\tau|=R_{1}} \frac{\left|\Psi^{\prime}(\tau)\right||d \tau|}{|\Psi(\tau)-\Psi(w)|^{2}}\left|\Psi^{\prime}(\tau)\right|=\frac{d(\Psi(\tau), L)}{|\tau|-1} . \\
& =\int_{\left\{|\tau|=R_{1}\right\} \cap \Delta_{1}} \frac{\left|\tau-w_{1}\right|^{\lambda_{1}-1-\varepsilon|d \tau|}}{|\tau-w|^{2\left(\lambda_{1}-\varepsilon\right)}}+\int_{\left\{|\tau|=R_{1}\right\} \cap \Delta_{2}} \frac{d(\Psi(\tau), L)|d \tau|}{(|\tau|-1)|\Psi(\tau)-\Psi(w)|^{2}} \\
& +\int_{\left\{|\tau|=R_{1}\right\} \cap\left(\widehat{\Delta}_{1} \cup \widehat{\Delta}_{2}\right)} \frac{(|\tau|-1)^{-\varepsilon}|d \tau|}{|\tau-w|^{2(1-\varepsilon)}} \\
& =: B_{n}^{1}(z)+B_{n}^{2}(z)+B_{n}^{3}(z) .
\end{aligned}
$$

Let us set:

$$
\begin{aligned}
& F_{1}:=\left\{\left\{|\tau|=R_{1}\right\} \cap \Delta_{1}:\left|\tau-w_{1}\right| \geq|\tau-w|\right\}, F_{2}:=\left\{\left\{|\tau|=R_{1}\right\} \cap \Delta_{2}:\left|\tau-w_{2}\right| \geq|\tau-w|\right\}, \\
& F_{3}:=\left(\left\{|\tau|=R_{1}\right\}\right) \backslash\left(F_{1} \cup F_{2}\right) .
\end{aligned}
$$

Under this notations we have:

$$
\begin{aligned}
& B_{n}^{1}(z)=\int_{F_{1}} \frac{\left|\tau-w_{1}\right|^{\lambda_{1}-1-\varepsilon}|d \tau|}{|\tau-w|^{2\left(\lambda_{1}+\varepsilon\right)}+\int_{F_{2}} \frac{\left|\tau-w_{1}\right|^{\lambda_{1}-1-\varepsilon}|d \tau|}{|\tau-w|^{2\left(\lambda_{1}+\varepsilon\right)}}} \\
& \prec \begin{cases}\left(\frac{1}{n}\right)^{\lambda_{1}-1-\varepsilon} \int_{F_{1}} \frac{|d \tau|}{|\tau-w|^{2\left(\lambda_{1}-\varepsilon\right)}}+\int_{F_{2}} \frac{|d \tau|}{|\tau-w|^{\lambda_{1}+1+\varepsilon}}, & \text { if } \lambda_{1} \geq 1, \\
\int_{F_{1}} \frac{|d \tau|}{|\tau-w|^{2\left(\lambda_{1}+\varepsilon\right)-\lambda_{1}+1-\varepsilon}}+\left(\frac{1}{n}\right)^{\lambda_{1}-1-\varepsilon} \int_{F_{2}} \frac{|d \tau|}{|\tau-w|^{2\left(\lambda_{1}+\varepsilon\right)},} & \text { if } \lambda_{1}<1,\end{cases} \\
& \prec\left\{\begin{array}{ll}
n^{\lambda_{1}+\varepsilon}, & \text { if } \lambda_{1} \geq 1, \\
n^{\lambda_{1}+\varepsilon}, & \text { if } \lambda_{1}<1,
\end{array}, \forall \varepsilon>0 ;\right. \\
& B_{n}^{2}(z)=\int_{\left\{|\tau|=R_{1}\right\} \cap\left(\widehat{\Delta}_{1} \cup \widehat{\Delta}_{2}\right)} \frac{d(\Psi(\tau), L)|d \tau|}{(|\tau|-1)|\Psi(\tau)-\Psi(w)|^{2}}<\left\{\begin{array}{ll}
n^{2}, & z \in \Omega(\delta), \\
n^{1+\varepsilon}, & z \in \widehat{\Omega}(\delta),
\end{array}, \forall \varepsilon>0 .\right. \\
& B_{n}^{3}(z)=\int_{\left\{|\tau|=R_{1}\right\} \cap \widehat{\Delta}_{1}} \frac{(|\tau|-1)^{-\varepsilon}|d \tau|}{|\tau-w|^{2(1-\varepsilon)}}<n^{1+\varepsilon}, \forall \varepsilon>0 .
\end{aligned}
$$

So, from (27), we have:

$$
B_{n}(z)<B_{n, 1}(z):=\left\{\begin{array}{ll}
n^{\widehat{\lambda}}, & z \in \Omega(\delta), \\
n^{1+\varepsilon}, & z \in \widehat{\Omega}(\delta),
\end{array}, \forall \varepsilon>0 .\right.
$$

Similarly, for the region $G \in C_{\theta}\left(\lambda_{1}, \lambda_{2}\right)$, we obtain: 


$$
B_{n}(z) \prec B_{n, 2}(z):=\left\{\begin{array}{ll}
n^{\tilde{\lambda}}, & z \in \Omega(\delta) \\
n^{1+\varepsilon}, & z \in \widehat{\Omega}(\delta),
\end{array}, \forall \varepsilon>0 .\right.
$$

Now, combining (16), (17), (25), (28) and (29) for the region, any $p>1, \gamma_{1}>-2,0<\lambda_{1}<2$, and for all sufficiently small $\varepsilon>0$,we obtain:

$$
\begin{gathered}
\left|P_{n}^{\prime}(z)\right|<|\Phi(z)|^{n+1}\left[\frac{1}{d\left(z, L_{R_{1}}\right)} \int_{L_{R_{1}}}\left|P_{n}(\zeta)\right| \frac{|d \zeta|}{|\zeta-z|}+\left|P_{n}(z)\right| \int_{L_{R_{1}}} \frac{|d \zeta|}{|\zeta-z|^{2}}\right] \\
<|\Phi(z)|^{n+1}\left\|P_{n}\right\|_{p}\left[\frac{1}{d\left(z, L_{R_{1}}\right)} G_{n, 1}(z)+\left|P_{n}(z)\right| B_{n, 1}(z)\right], \text { if } G \in C_{\theta}\left(\lambda_{1}, 2\right), \\
\left|P_{n}^{\prime}(z)\right|<|\Phi(z)|^{n+1}\left\|P_{n}\right\|_{p}\left[\frac{1}{d\left(z, L_{R_{1}}\right)} G_{n, 2}(z)+\left|P_{n}(z)\right| B_{n, 2}(z)\right], \text { if } G \in C_{\theta}\left(\lambda_{1}, \lambda_{2}\right),
\end{gathered}
$$

Now, using estimates for $\left|P_{n}(z)\right|$ ([25, Theorem 1 and Corollary 1]) for the cases $G \in C_{\theta}\left(\lambda_{1}, 2\right)$ and $G \in C_{\theta}\left(\lambda_{1}, \lambda_{2}\right)$, we get:

and

$$
\left|P_{n}^{\prime}(z)\right| \prec\left\|P_{n}\right\|_{p}\left[\frac{|\Phi(z)|^{n+1}}{d\left(z, L_{R_{1}}\right)} G_{n, 1}(z)+\frac{|\Phi(z)|^{2(n+1)}}{d^{\frac{2}{p}}\left(z, L_{1+\frac{1}{n}}\right)} E_{n, 1} B_{n, 1}(z)\right] \text {, if } G \in C_{\theta}\left(\lambda_{1}, 2\right),
$$

where

$$
\left|P_{n}^{\prime}(z)\right| \prec\left\|P_{n}\right\|_{p}\left[\frac{|\Phi(z)|^{n+1}}{d\left(z, L_{R_{1}}\right)} G_{n, 2}(z)+\frac{|\Phi(z)|^{2(n+1)}}{d^{\frac{2}{p}}\left(z, L_{1+\frac{1}{n}}\right)} E_{n, 2} B_{n, 2}(z)\right] \text {, if } G \in C_{\theta}\left(\lambda_{1}, \lambda_{2}\right),
$$

$$
\begin{aligned}
& E_{n ; 1}:=\left\{\begin{array}{ll}
n^{\frac{\tilde{\gamma} \cdot \hat{\lambda}}{p}}, & \text { if } \tilde{\gamma} \cdot \hat{\lambda} \geq 1, \\
n^{\frac{1}{p},} & \text { if } \tilde{\gamma} \cdot \hat{\lambda}<1,
\end{array} \quad E_{n, 2}:= \begin{cases}n^{\frac{\tilde{\gamma} \cdot \tilde{\lambda}}{p},} & \text { if } \tilde{\gamma} \cdot \tilde{\lambda} \geq 1, \\
n^{\frac{1}{p},} & \text { if } \tilde{\gamma} \cdot \tilde{\lambda}<1,\end{cases} \right. \\
& \hat{\lambda}:=\left\{\begin{array}{ll}
\max \{1 ; \lambda\}+\varepsilon, & \text { if } 0<\lambda<2, \\
2, & \text { if } \lambda=2,
\end{array} ; \quad \begin{array}{ll}
\tilde{\gamma}_{1}, & \text { if } 0<\lambda<2, \\
\tilde{\gamma}_{2}, & \text { if } \lambda=2,
\end{array}\right. \\
& \tilde{\gamma}_{i}:=\max \left\{0 ; \gamma_{i}\right\}, i=1,2 ; \tilde{\lambda}:=\max \left\{1 ; \lambda_{1}, \lambda_{2}\right\}+\varepsilon \text {. }
\end{aligned}
$$

Therefore, we complete the proof of Theorems 5 and 6.

\section{References}

[1] Abdullayev F. G., Andrievskii V. V., "On the orthogonal polynomials in the domains with K-quasiconformal boundary”, Izv. Akad. Nauk Azerb. SSR., Ser. FTM. 1, (1983), 3-7.

[2] Abdullayev F. G., "On the interference of the weight boundary contour for orthogonal polynomials over the region", J. of Comp. Anal. and Appl., 6 (1), (2004), 31-42.

[3] Abdullayev F. G., Aral D., "On the Bernstein-Walsh type Lemmas in regions of the complex plane", Ukr. Math. J., Vol. 63 (3), (2011), 337-350.

[4] Abdullayev F. G., Özkartepe P., "An analogue of the Bernstein-Walsh lemma in Jordan regions of the complex plane”, Journal Ineq. and Appl., 2013:570 (2013), 7 pp.

[5] Abdullayev F. G., Gün C. D., "On the behavior of the algebraic polynomials in regions with piecewise smooth boundary without cusps", Ann. Polon. Math., 111, (2014), 39-58.

[6] Abdullayev F. G., Özkartepe P., "On the behavior of the algebraic polynomial in unbounded regions with piecewise Dini-smooth boundary”, Ukr. Math. J. , 66 (5), (2015), 645-665.

[7] Abdullayev F. G., Gün C. D., Özkartepe P., "Inequalities for algebraic polynomials in regions with exterior cusps”, J. Nonlinear Funct. Anal. 3, (2015), 1-32. 
[8] Abdullayev F. G., Özkartepe P., "On the growth of algebraic polynomials in the whole complex plane”, J. Korean Math. Soc. 52 (4), (2015), 699-725.

[9] Abdullayev F. G., Özkartepe P., "Uniform and pointwise polynomial inequalities in regions with cusps in the weighted Lebesgue space", Jaen Journal on Approximation, 7 (2), (2015), 231-261.

[10] Abdullayev F. G., Özkartepe P., "Polynomial inequalities in Lavrentiev regions with interior and exterior zero angles in the weighted Lebesgue space", Publications de l'Institut Mathématique (Beograd), 100 (114), (2016), 209-227.

[11] Abdullayev F. G., Özkartepe N.P., "Uniform and pointwise Bernstein-Walsh-type inequalities on a quasidisk in the complex plane”, Bull. Belg. Math. Soc., 23 (2), (2016), 285-310.

[12] Abdullayev F. G., Gün C. D., "Bernstein-Walsh -type inequalities for derivatives of algebraic polynomials", 2020. (to appear)

[13] Ahlfors L., "Lectures on quasiconformal mappings", Princeton, NJ: Van Nostrand, 1966.

[14] Andrievskii V. V., Belyi V. I., Dzyadyk V. K., "Conformal invariants in cocstructive theory of functions of complex plane”, Atlanta, World Federatin Publ. Com., 1995.

[15] Andrievskii V. V., "Weighted polynomial inequalities in the complex plane”, J. Approx.Theory, 164 (9), (2012), 1165-1183.

[16] Belinskii P. P., “General properties of quasiconformal mappings”, Nauka, Sib. otd., Novosibirsk, 1974. [in Russian]

[17]Dzjadyk V. K., “Introduction to the theory of uniform approximation of function by polynomials", Nauka, Moskow, 1977.

[18] Gaier D., "On the convergence of the Bieberbach polynomials in regions with corners", Constructive Approximation, 4, (1988), 289-305.

[19]Hille E., Szegö G., Tamarkin J. D., “On some generalization of a theorem of A.Markoff”, Duke Math. J., 3 (1937), 729-739.

[20]Lehto O., Virtanen K.I., "Quasiconformal mapping in the plane”, Springer Verlag, Berlin, 1973.

[21] Mergelyan S. N., "Some questions of constructive functions theory", Proc. of the Steklov Institute of Mathematics, Vol. XXXVII, 1951, 1-92. [in Russian]

[22] Özkartepe P., "Pointwise Bernstein-Walsh-type inequalities in regions with piecewise Dini-smooth boundary", MJEN, 5 (3), (2017), 35-47.

[23] Rickman S., "Characterisation of quasiconformal arcs", Ann. Acad. Sci. Fenn., Ser. A, Mathematica., 395, (1966), $30 \mathrm{pp}$.

[24] Stylianopoulos N., "Strong asymptotics for Bergman polynomials over domains with corners and applications", Const. Approx., 38 (1), (2012), 59-100.

[25] Tunc T., Şimşek D., Oruç E., "Pointwise Bernstein-Walsh-type inequalities in regions with interior zero angles in the Bergman space”, Trans. of NAS of Azerbaijan Ser. of Phys.-Tech. and Math. Sciences, 37 (1), (2017), 169-182.

[26] Walsh J. L., "Interpolation and approximation by rational functions in the complex domain", AMS, 1960.

[27] Warschawski S. E., "Über das Randverhalten der Ableitung der Abbildungsfunktion bei konformer Abbildung”, Math.Z., 35, (1932), 321-456. 